\title{
On the Preon Model
}

\author{
Hesham Mansour \\ Physics Department, Faculty of Science, Cairo University, Giza, Egypt \\ Email: hesham@sci.cu.edu.eg
}

How to cite this paper: Mansour, $\mathrm{H}$. (2019) On the Preon Model. Open Journal of Microphysics, 9, 11-14.

https://doi.org/10.4236/ojm.2019.92002

Received: March 25, 2019

Accepted: May 13, 2019

Published: May 16, 2019

Copyright $\odot 2019$ by author(s) and Scientific Research Publishing Inc. This work is licensed under the Creative Commons Attribution International License (CC BY 4.0).

http://creativecommons.org/licenses/by/4.0/

\begin{abstract}
One of the fundamental questions is that "what the matter is composed of?" In 1897, atoms are known as the basic building blocks of matter. In the year 1911, Ernest Rutherford demonstrated that when alpha particles are scattered on a thin gold foil that the atom is composed of mostly empty space with a dense core at its center which is called the nucleus. Thereafter, protons and neutrons were discovered. In 1956, McAllister and Hofstadter published experimental results of elastic scattering of the electrons from a hydrogen target which revealed that the proton has an internal structure. In 1964, Gell-Mann (and independently) Zweig proposed that nucleons are composed of point-like particles which are called quarks. These quarks are postulated to have spin-1/2, fractional electric charge. Combinations of different flavors of quarks yield protons and neutrons which belong to the type of particles called baryons (built up from three quarks) and mesons as (quark and an antiquark). These two groups of particles are categorized as hadrons. The quarks showed further decay properties which suggested that they have a substructure.
\end{abstract}

\section{Keywords}

Quark Model, Preon Model, Building Blocks

\section{Introduction}

The fact that fundamental particles have quark substructures was introduced in 1964, following the success of the quark model and its decay modes. It was assumed that the quarks have substructures called the preons [1] [2]. Several preon models were proposed [3]-[11] to explain the Standard Model SM, predicting small discrepancies with such a model and generating new particles and certain phenomena, which are outside the Standard Model. The preon models were introduced among other things to mainly reduce a large number of particles, many that differ only in charge, to a smaller number of more fundamental particles. The huge number of particles was referred to as the "particle zoo". In the Stan- 
dard Model, there are classes of particles. e.g. the quarks, which have six types, of which there are three varieties in each "colors", red, green, and blue, giving rise to chromodynamics. Additionally, there are six different types which are known as leptons. Of these six leptons, there are three particles: the electron, muon, and tau. The neutrinos comprise the other three leptons, and for each neutrino, there is a corresponding member from the other set of the three leptons. The SM generally predicts equal amounts of matter and antimatter in the universe which has not yet been solved. Preon models are motivated by a desire to replicate the achievements of the periodic table, i.e. to reduce the particles to a few building blocks. Preon models assumed additional unobserved forces or dynamics to account for the observed properties of elementary particles, which conflicted with the experimental observations. In the next section, we present criticism of a recently published toy preon model [12].

\section{The Preon Model}

The work in Ref. [12] proposes (I quote) a preon model derived from a first-order mass formula. The preon masses are derived from quark masses, and the model assumes that six preons (D, U, S, C, B, and T) form the six known quarks ( $\mathrm{d}, \mathrm{u}, \mathrm{s}, \mathrm{c}, \mathrm{b}$, and $\mathrm{t}$ ). A few assumptions were made to formulate the model. The proposed preon model assumes that each quark is composed of three spin $1 / 2$ preons. In its ground state, a quark has zero total orbital angular momentum and total angular momentum of 1/2. Preon masses are derived from a first-order mass formula that was based on the work of Zel'dovich and Sakharov [13] [14] who proposed semiempirical mass formulas that provide predictions for mesons and baryons in terms of effective quark masses. Within this formulation, quark wave functions are assumed to reside in their lowest $1 \mathrm{~S}$ state. In a similar manner, the first-order mass formula has been applied in Ref. [12] to predict the preon masses based on the quark masses. In this approach, a quark mass $M_{i}$ is given by

$$
\begin{aligned}
M_{i}= & \delta+\sum_{j=1}^{3} m_{j}^{i}+\xi / 3\left[m_{0}^{2} /\left(m_{1}^{i} m_{2}^{i}\right) \sigma_{1} \cdot \boldsymbol{\sigma}_{2}+m_{0}^{2} /\left(m_{1}^{i} m_{3}^{i}\right) \sigma_{1} \cdot \boldsymbol{\sigma}_{3}\right. \\
& \left.+m_{0}^{2} /\left(m_{2}^{i} m_{3}^{i}\right) \sigma_{2} \cdot \boldsymbol{\sigma}_{3}\right]
\end{aligned}
$$

where $\delta$ and $\xi$ are parameters to be determined, $\mathrm{m}_{\mathrm{j}}^{\mathrm{i}}$ labels the ith preon type (i.e., $\mathrm{D}, \mathrm{U}, \mathrm{S}, \mathrm{C}, \mathrm{B}$, and $\mathrm{T})$ for the three preons $(j=1,2$, and 3$)$ that comprises the quark, $m_{0}$ is the average mass of first generation preons ( $\mathrm{D}$ and $\left.\mathrm{U}\right)$, and $\sigma_{k}(k=1$, 2 , and 3 ) is the spin vectors for the preons incorporated into the quark. The last term in Equation (1) represents the spin-spin interaction of the preons and $\sigma_{k} \cdot \sigma_{1}$ has the values of $-3 / 4$ and $+1 / 4$ for singlet and triplet composite state, respectively. The last sentence which is wrong is repeated after Equation (1) by the same author in Ref. [15]. In fact, the spin of the three particles has a sum which is equal to the spin of the compound quark, which is not given in the tabulated results of Ref. [12]. The correct spin products are really given in Ref. [14]. Equation (1) is solved for the preon masses given the quark masses. The 
preons are arranged in three generations: $[D, U],[S, C]$, and $[B, T]$. These six preon types are used in Equation (1), and are assumed to have the following spin and charge assignments (i.e., D (1/2, -e/9), U (1/2, +2e/9), S (1/2, -e/9), C (1/2, $+2 \mathrm{e} / 9), \mathrm{B}(1 / 2,-\mathrm{e} / 9), \mathrm{T}(1 / 2,+2 \mathrm{e} / 9))$. In order to determine the preon masses, it is necessary to impose a few assumptions. The following assumptions are made to specify the proposed preon model:

- The quarks are comprised of preons of the same type. For example, $u$ quark is defined by $3 \mathrm{U}$ preons and $s$ quark is comprised of $3 \mathrm{~S}$ preons.

- There are 6 preons types: D, U, S, C, B, and T.

- The preons are in a relative $S$ state in the mass formula of Equation (1). The mass (m) of the $\mathrm{U}$ preon is derived from the $u \rightarrow d+W^{+}$vertex, but the mass of the $d$ quark is ignored in Equation (2):

$$
m(\mathrm{U})=M\left(W^{+}\right) / 3
$$

Here again Equation (2) is wrong for two reasons:

Relativistic kinematics should be used i.e. for a decay of one particle $M$,

$$
\begin{gathered}
0 \rightarrow 1+2 \\
M^{2}=-M_{2}^{2}+2 M E_{2}+M_{1}^{2}
\end{gathered}
$$

in the rest frame of particle 0 .

Also, Equation (2) means that the three particles are not bound because of the division by 3 .

- The mass of the $\mathrm{D}$ preon is related to the $\mathrm{U}$ preon and $u$ and $d$ quark masses as follows:

$$
m(\mathrm{D})=\left(M_{d} / M_{u}\right) m(\mathrm{U})
$$

- The $\delta$ and $\xi$ parameters are determined from Equation (1) using the $\mathrm{U}$ and D preon masses.

- The quantity $m_{0}$ is defined in a manner that is analogous to the first-order quark mass formula [14].

$$
m_{0}=(m(\mathrm{U})+m(\mathrm{D})) / 2
$$

- The S, C, B, and T preon masses are determined from Equation (1), the U and $\mathrm{D}$ preon masses, the masses of $s, c, b$, and $t$ quarks, and the $\delta$ and $\xi$ parameters.

\section{Conclusion}

The fact that the quark quark forces differ from the preon forces as well as from the nuclear forces in their shapes and strengths suggests that one cannot import structure models from one system to another. Hence, one concludes that the model with the above previously reported mistakes is not valid and the tabulated data are undoubtedly erroneous and incomplete.

\section{Conflicts of Interest}

The authors declare no conflicts of interest regarding the publication of this paper. 


\section{References}

[1] Gell-Mann, M. (1964) A Schematic Model of Baryons and Mesons. Physics Letters, 8, 214-215. https://doi.org/10.1016/S0031-9163(64)92001-3

[2] Pati, J. and Salam, A. (1974) Lepton Number as the Fourth "Color". Physical Review D, 10, 275-289. https://doi.org/10.1103/PhysRevD.10.275

[3] Terazawa, H., Chikashige, Y. and Akama, K. (1977) Unified Model of the Nambu-Jona-Lasinio Type for All Elementary-Particle Forces. Physical Review D, 15, 480-487. https://doi.org/10.1103/PhysRevD.15.480

[4] Harari, H. (1979) A Schematic Model of Quarks and Leptons. Physics Letters B, 86, 83-86. https://doi.org/10.1016/0370-2693(79)90626-9

[5] Shupe, M. (1979) A Composite Model of Leptons and Quarks. Physics Letters B, 86, 87-92. https://doi.org/10.1016/0370-2693(79)90627-0

[6] D’Souza, I.A. and Kalman, C.S. (1992) Preons: Models of Leptons, Quarks, and Gauge Bosons as Composite Objects. World Scientific Publishing, Hong Kong. https://doi.org/10.1142/9789814354769

[7] Bilson-Thompson, S., Hackett, J., Kauffman, L. and Wan, Y.D. (2012) Emergent Braided Matter of Quantum Geometry. Symmetry, Integrability and Geometry: Methods and Applications, 8, 1-43.

[8] Bevelacqua, J.J. (2012) Physical Interpretation of Electrodynamics within the Yang-Mills Theory. Physics Essays, 25, 369-373. https://doi.org/10.4006/0836-1398-25.3.369

[9] Żenczykowski, P. (2016) The Harari-Shupe Observation without Preons-A Glimpse of Physics to Come? Acta Physica Polonica B, 47, 1011-1032. https://doi.org/10.5506/APhysPolB.47.1011

[10] Larson, D.L. (2017) Additional Evidence and Predictions for the ABC Preon Model. Physics Essays, 30, 347-355. https://doi.org/10.4006/0836-1398-30.4.347

[11] Raitio, R. (2017) Preon Model, Knot Algebra and Gravity. Open Access Library Journal, 4, e3432.

[12] Bevelacqua, J.J. (2019) A First-Order Mass Formula for Quarks in Terms of Constituent Preons. Journal of Nuclear and Particle Physics, 9, 1-4.

[13] Zel'dovich, Y.B. and Sakharov, A.D. (1966) The Quark Structure and Masses of Strongly Interacting Particles. Yadernaya Fizika, 4, 395-406.

[14] Sakharov, A.D. (1980) Mass Formula for Mesons and Baryons. Journal of Experimental and Theoretical Physics, 51, 1059-1060.

[15] Bevelacqua, J.J. (2018) Description of Selected Hexaquark States in Terms of a First-Order Mass Formula. Physics Essays, 31, 104-107.

https://doi.org/10.4006/0836-1398-31.1.104 